\title{
Application of Homography Transformation for Assisting Machine Vision in Automatic Guided Vehicles
}

\author{
Ching-Wei Lee ${ }^{1 *}$, Yi-Chan Pei ${ }^{1}$, Kuo-Shen Chen ${ }^{2}$, Sen-Yung Lee ${ }^{2}$ \\ ${ }^{1}$ Graduate Student, ${ }^{2}$ Professor \\ Department of Mechanical Engineering, National Cheng-Kung University, \\ No.1, Daxue Rd., East Dist., Tainan City 701, Taiwan \\ *Corresponding Author: weiwei51415@gmail.com
}

\begin{abstract}
The safety of autonomous car driving is an important concern raised in the recent years. With the development of various sensors, lots of driving assistant device are applied in the vehicle, such as sonar sensing and inertial navigation Other than terms listed above, machine vision is also a momentous technology in the automatic guided vehicle. Automatic guided vehicle integrates technologies such as machine vision and automatic control, and expects to use computer-assisted driving to enhance the safety of driving. However, the location of the camera installation limited by car structures could cause the camera cannot properly record the road situation at the optimal observation point, and the image could be significantly distorted. Therefore, the image recorded by camera have to be corrected in order to reduce the image distortion. This study utilizes a webcam to obtain image of the surrounding, and process the image with LabVIEW for promote the precision of geometric matching via homography transformation. Meanwhile, an automatic guided vehicle is also realized by hiring IG-42 motors and using a MyRIO board and LabVIEW as the program control and the central information hub. After integrating the perspective calibration for geometric matching and automatic guided vehicle, we conducted an experiment of tracking a mark line, which is commonly used in manufacturing factories. In addition, we also tested the geometric matching of the image after correction. The distinguish rates rose from 0.63 to 0.93 , represents uncorrected and corrected respectively. Finally, we finish an automatic guided vehicle by machine vision. A more complete system will go on in the future.
\end{abstract}

Keywords: Homography, Perspective calibration, automatic Guided Vehicle

\section{Introduction}

Autonomous guided vehicles (AGV) and their navigations are extremely important [1] in factory managements, logistics, and other assembly automations. In recent years, with the advanced in artificial intelligence, automatic self-driving cars becomes potentially a valuable target for next generation car industry. In both cases, driving safety is a key concerns. Although there are many devices to prevent the accidents happen, mostly car accidents occur from the inattention of driver and the blind areas of the car driving. Limited by the viewable scope from the car, driver cannot get the full conditions around the car. For the reason that, there are many cameras be installed in the car in order to get the more perspectives on nearby environment. However, this is difficult to get a view with a preferred attitude. The location of the camera installation limited by the available structure and geometry of cars. Nevertheless, it is possible to utilize the perspective calibration algorithm to convert distorted images to a less distortive ones. Therefore, lots of vehicle industries propose related improvements to expand the field of driving view, such as Nissan $360^{\circ}$ vision assistant of the AVM (Around View Monitoring System) [2], which make drivers to observe the surrounding more easily. With four cameras around the car and combining the perspective transformation and image processing, drivers can get the road condition more directly from the top view. This is not only enhancing the field of view but also improving the safety of driving. Although the function of perspective calibration has been wide-spread 
used, with adding homography transformation, user can convert images into different perspectives [3] which they required and thus would significantly further improving the ability to judge environmental obstacles. User can get the information more intuitively, on the other hand, they can increase the ability of analyze the driving situation.

In previous works, the camera is usually mounted on the roof simulate the driver's sight. Maybe the stability of the vehicle can be promoted and the sign on the road surface can be more clearly observed if one uses the perspective transformation to obtain undistorted vision from the top view of a car. For verifying the assumption and for promoting the future applications, the goal of this work is to integrate the perspective calibration, vision line tracking, and the automatic guided vehicle to establish a comprehensive system. In order to achieve the goal, we need build a real time perspective calibration and image detection system. Meanwhile, a homemade prototype will be built to validate the simulation of homography in practice by automatic guided vehicle with machine vision. Fig. 2 shows the research flow of this work which presents the overall plan in machine vision processing and experimental validation. It is believed that by the effort of this work, not only an automatic guided vehicle prototype will be established, but an effective vision assistant system will also be set up. Both should make significant contributions in machine vision development and a more complicated system will be realized in the near future.

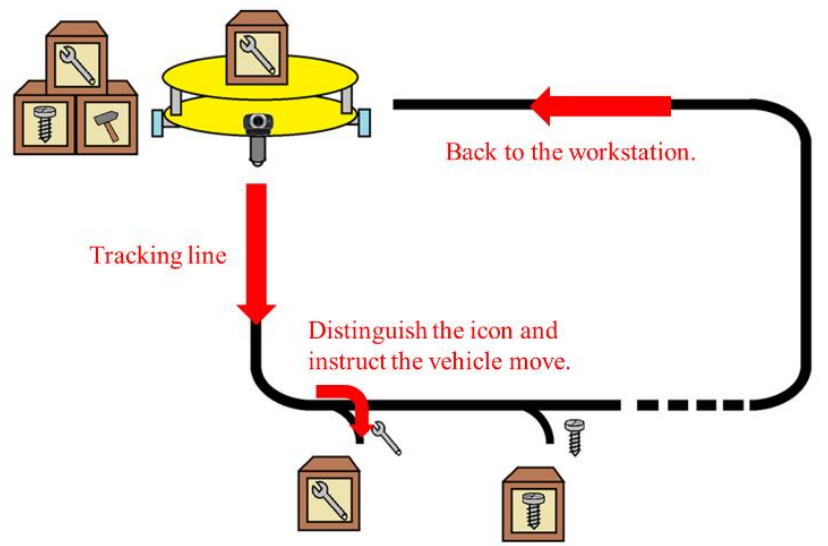

Fig.1 unmanned transportation and classification system

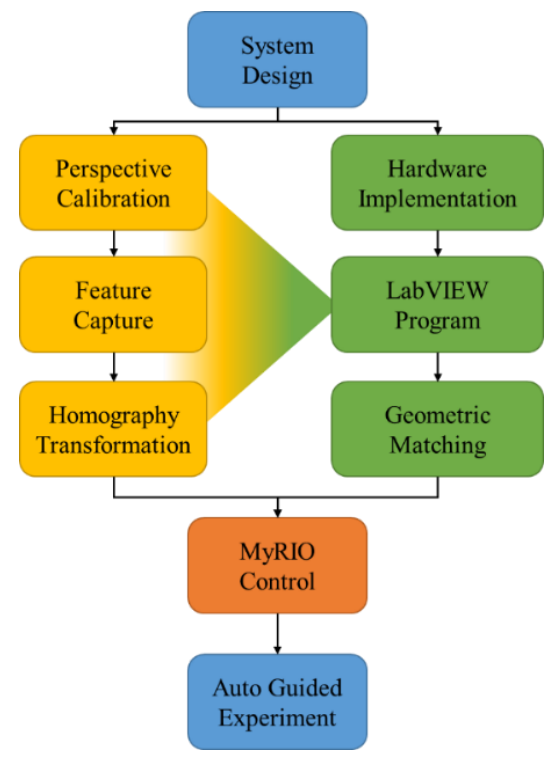

Fig. 2 Research flow

\section{Perspective Calibration}

This section describes the principle of perspective calibration using a homography matrix. It also explains the approach which the four feature points required to calculate the homography matrix are captured in this experiment.

\subsection{Introduction Homography}

Image process is a great approach to enhance the accuracy of machine vision [4]. If the image is used directly without image processing, features of the object which is observed would not be clearly capture. The homography transforms is a concept of transformation in geometry [5]. When the two images, $\mathrm{A}$ and $\mathrm{B}$, are obtained from the different perspective with the same object, we can set the homography matrix $\boldsymbol{H}$. Multiply the image A matrix by $\boldsymbol{H}$ convert it into image $\mathrm{B}$. The homography transformation is to convert the pixel coordinate $Q(x, y)$ of the object which acquired in the image $\mathrm{A}$ into the pixel coordinate $q(u, v)$ of the same object in the image B, as show in Fig.3.

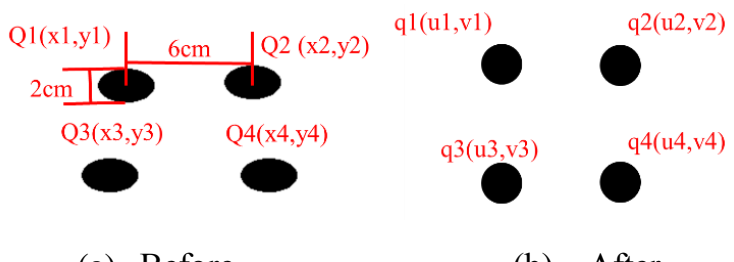
(a) Before
(b) After

Fig.3 Homography transformation 
The two coordinates can be expressed as:

$$
Q=\left[\begin{array}{l}
x \\
y \\
1
\end{array}\right], q=\left[\begin{array}{l}
u \\
v \\
1
\end{array}\right], q=H * Q
$$

Where $H$ is expressed as:

$$
\left[\begin{array}{l}
\mathrm{u} \\
\mathrm{v} \\
1
\end{array}\right]=\left[\begin{array}{llc}
\mathrm{h}_{11} & \mathrm{~h}_{12} & \mathrm{~h}_{13} \\
\mathrm{~h}_{21} & \mathrm{~h}_{22} & \mathrm{~h}_{23} \\
\mathrm{~h}_{31} & \mathrm{~h}_{32} & 1
\end{array}\right]\left[\begin{array}{l}
x \\
y \\
1
\end{array}\right]
$$

The matrix $h$ is a matrix with 8 degrees of freedom. Therefore, at least 8 equations are required to solve the matrix. Furthermore, we need four points form the image to calculate the solution of 8 unknown variables.

\subsection{Capture Calibration Points}

In order to achieve the perspective calibration, we must obtain the eight parameters of the homography matrix by grabbing the four feature points in the image. So we built a vision system that automatically grabbed four feature points. With image processing and square dot pattern, the system can automatically capture four feature points to convert the image from the camera into a top view. The simple square dot pattern has the following specifications. The center of each of the four points is $6 \mathrm{~cm}$ apart, and the radius of each dot is $1 \mathrm{~cm}$, as shown in Fig. 3(a).

Use the threshold to split the image into foreground and background (Fig. 4(a)), record all the $\mathrm{x}$ and $\mathrm{y}$ coordinates $\left(x_{i}, y_{i}\right)$ of the foreground, and calculate the average $(\bar{x}, \bar{y})$, we can roughly get the center position of 4 points. It is important to note that the center position here is not the center of the point, but four points of the diagonal intersecting lines. By obtaining the center position $(\bar{x}, \bar{y})$ of this point, compared with the coordinates of all the points recorded $\left(x_{i}, y_{i}\right)$, it can be divided into 4 categories, as shown in Table 1:

Table 1. feature categories

\begin{tabular}{|c|c|}
\hline$x_{i}<\bar{x}, y_{i}<\bar{y}$ & $\mathrm{P} 1$ \\
\hline$x_{i}>\bar{x}, y_{i}<\bar{y}$ & $\mathrm{P} 2$ \\
\hline$x_{i}<\bar{x}, y_{i}>\bar{y}$ & $\mathrm{P} 3$ \\
\hline$x_{i}>\bar{x}, y_{i}>\bar{y}$ & $\mathrm{P} 4$ \\
\hline
\end{tabular}

However, since more than 10,000 points are recorded, and when an unknown number of coordinates are recorded, a dynamic matrix must be used to record all coordinates, thus resulting in a very slow calculation. Therefore, in order to reduce the coordinate point of the record, use Laplace's $3 * 3$ mask and image convolution to find the edge of the square dot, and then record the $\mathrm{x}$ and $\mathrm{y}$ coordinates of the boundary point (Fig. 4(b)) to increase the operation speed.

After completing the Laplace mask convolution and feature classification, we calculate the average of the $\mathrm{x}$ and $\mathrm{y}$ coordinates of each category, which is the coordinate position of the 4 feature points.

\section{Simulation}

This section uses LabVIEW programming to achieve homography matrix transformation. And we also conducted experiments to prove that the perspective calibration can improve the ability of geometric matching.

\subsection{Perspective Calibration Test}

On the basis of previous development, it is possible to utilize LabVIEW to put the perspective calibration in practical. After use threshold calculation and Laplace mask convolution to the image, as shown in Fig.4, we classify according to Table 1 and calculate the position of the four feature points. Then use the formulas (1) to (2) in Section 2.1 to calculate the homography matrix, and use this matrix to correct the image perspective, as shown in Fig.4 (c).

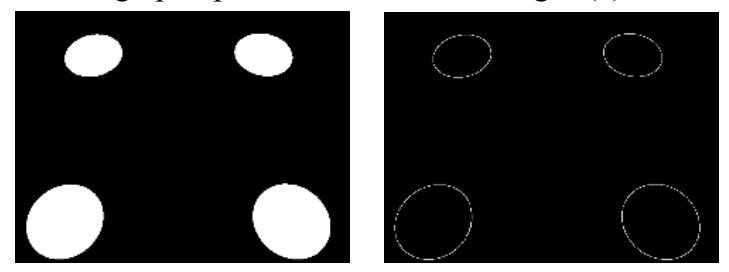

(a) threshold $\quad$ (b)Laplace mask convolution

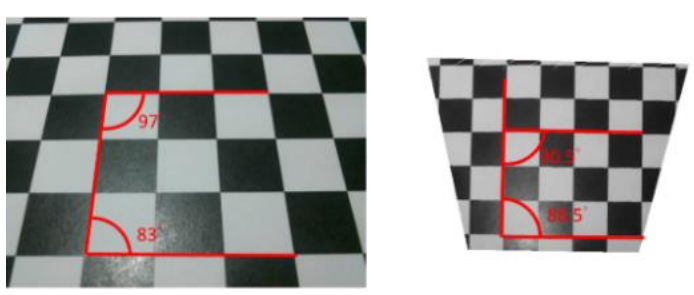

(c)checkerboard

Fig.4 experimental diagram 
Through the test of the checkerboard, it can be estimated that the results of the visual correction are within $2^{\circ}$ of the error. However, due to the decrease in resolution, some disadvantages are generated after image correction. As shown in Fig. 4(c) right side, the white point is guided on the image due to insufficient resolution of the input image. This shortcoming can be removed by median filtering and the output of the complete picture. The median filtering method is a statistically ordered filter. For a pixel of the image, median filtering sorts all pixels in the mask range and replaces the current pixel value with the median.

\subsection{Perspective Calibration for Geometric Matching}

After validating the perspective calibration simulation, we use the method to assist conduct the Geometric matching test. Geometric matching use geometric information to find images that are similar to the template of the image. The process of geometric matching is as follows. First, create a template of the object which is the target and calculate the geometric features. The program searches for areas of the image that are similar to the template and Calculate the similarity of these areas and quantify the score. We use the geometry matching module in LabVIEW for programming, and we can get the position and score from the module. In this way, we can classify objects and use this function to achieve automatic classification goals in fully automated transportation. We conduct the following experiments. Fix the camera and the image so we can know the correct position of the image in the camera. We use the coordinate position as the criterion for judging whether the geometric matching is correct, and use the correct position coordinates $(x \pm 10, y \pm 10)$ as the range. Use the program to calculate the detection times, detected object times, correct detected object times, and wrong detected object times. The result is as shown in Table 2. We define the success rates are correct detect object times divided by detection times. The success rates rose from 0.63 to 0.93 , represents uncorrected and corrected respectively.

With the homography matrix correction, the image recognition can be improved and the correct recognition rate can be improved, as shown in Fig.5. However, we have not used this method to capture four features for homography matrix but use the method in Section 2.2. This is because the geometric matching does not correctly capture the target feature for the uncorrected image. This makes it difficult to convert the perspective correctly. In addition to improving the accuracy of geometric matching, the perspective calibration can also correct the position information. From Fig. 6, we can see that the unit distance in the y direction before calibration will be smaller as the actual distance is further away. After the perspective calibration is work, the unit length can be corrected.

Table. 2 Homography transformation for geometric matching test

\begin{tabular}{|c|c|c|c|c|c|}
\hline & item & Test 1 & Test 2 & Test 3 & avg \\
\hline & detection & 1730 & 1705 & 1709 & 1714.6 \\
\hline \multirow{3}{*}{$\begin{array}{c}\text { Before } \\
\text { Perspective } \\
\text { calibration }\end{array}$} & detect object & 780 & 1177 & 1300 & 1085.6 \\
\hline & $\begin{array}{c}\text { correct detect } \\
\text { object }\end{array}$ & 298 & 1133 & 723 & 718 \\
\hline & $\begin{array}{l}\text { wrong detect } \\
\text { object }\end{array}$ & 482 & 44 & 577 & 367.6 \\
\hline \multirow{3}{*}{$\begin{array}{c}\text { After } \\
\text { Perspective } \\
\text { calibration }\end{array}$} & detect object & 1185 & 1634 & 1680 & 1499.6 \\
\hline & $\begin{array}{c}\text { correct detect } \\
\text { object }\end{array}$ & 1023 & 1566 & 1644 & 1411 \\
\hline & $\begin{array}{c}\text { wrong detect } \\
\text { object }\end{array}$ & 162 & 68 & 36 & 88.6 \\
\hline
\end{tabular}
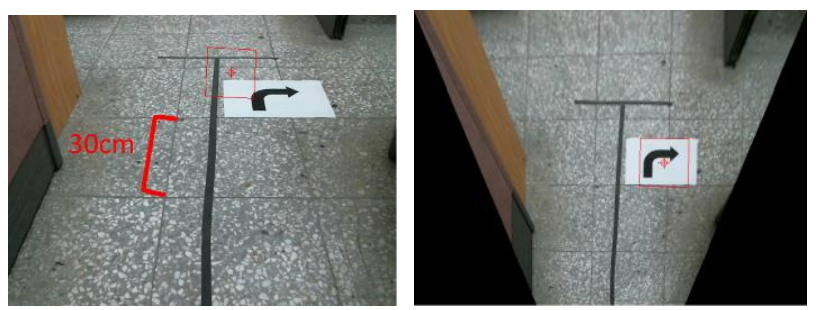

Fig.5 After perspective calibration can be recognized
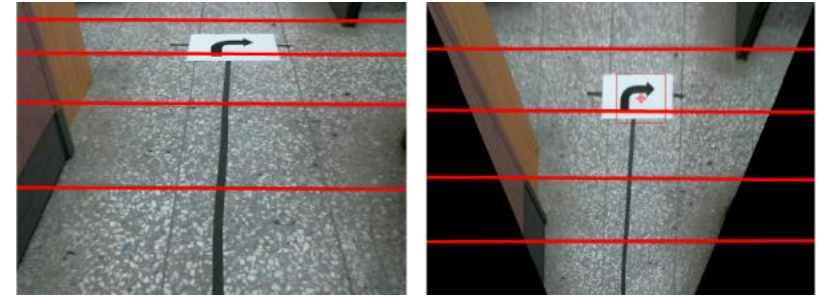

Fig.6 After perspective calibration correct unit length 


\section{Experimental Validation and Demonstration}

\subsection{Setup}

In order to implement the prototype of the automatic guided vehicle efficiently, as show in Fig.7, two IG-42 motors are used as the actuator and two auxiliary wheels for balance. A MyRIO-1900 board is used to control the two HB25 motor controller. By typing the speed and curve, the control board can calculate the motor control parametric and send the corresponding PWM signal to the HB-25. This makes it possible to realize the vision line tracking test with the relative position between the target line and the vehicle. The relative position is utilizing the camera which installed on the vehicle to capture the target line position in the image. This scheme utilizes Logitech HD C615 webcam, which can provide resolution of $1920 * 1080,640 * 480,320 * 240$, etc. And it can connect with MyRIO control board to provide image input for automatic guided vehicle.

\subsection{Software Interface}

LabVIEW is used for receiving and analyzing data from the webcam and instructing MyRIO and HB-25. We can manipulate the vehicle by the joystick, which is designed to prevent the car from getting out of control. We can dominate the car through the switching action manual mode, besides the automatic mode by vision guided can be activated. Meanwhile, LabVIEW interface can also monitor and adjust parameters.

\subsection{Vision Line Tracking Demo}

By integrating perspective correction, machine vision recognition and remote control vehicle, we build an automatic guided vehicle based on machine vision. We used black tape as the target line and used LabVIEW machine vision assistant to determine the position and slope of the target line. After grabbing the target line, we use the PID control method to control the moving of the vision automatic guided vehicle. The $\mathrm{x}$ coordinate 160 of the window center (resolution $320 * 240$ ) is used as the setpoint of the PID control, and the target line center is used as the process variable for PID control. After completing the vision line tracking test, we put a horizontal line on the head and tail of the line. And let the vehicle automatically return when it senses the horizontal line.

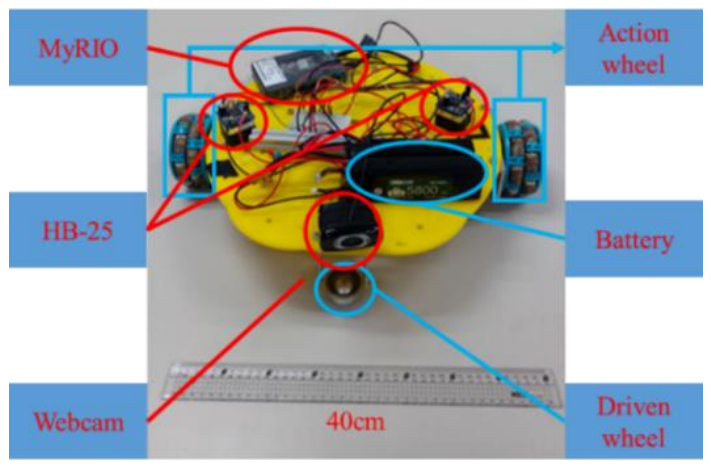

Fig.7 Automatic guided vehicle

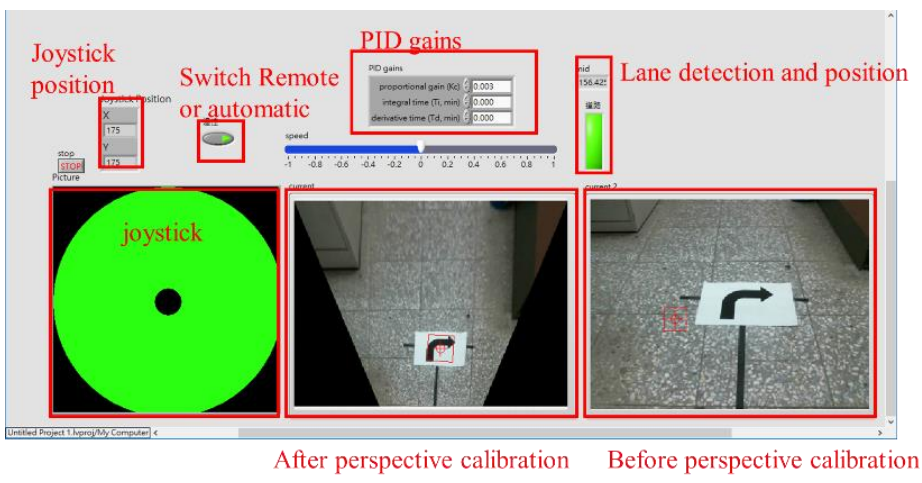

Fig.8 LabVIEW interface
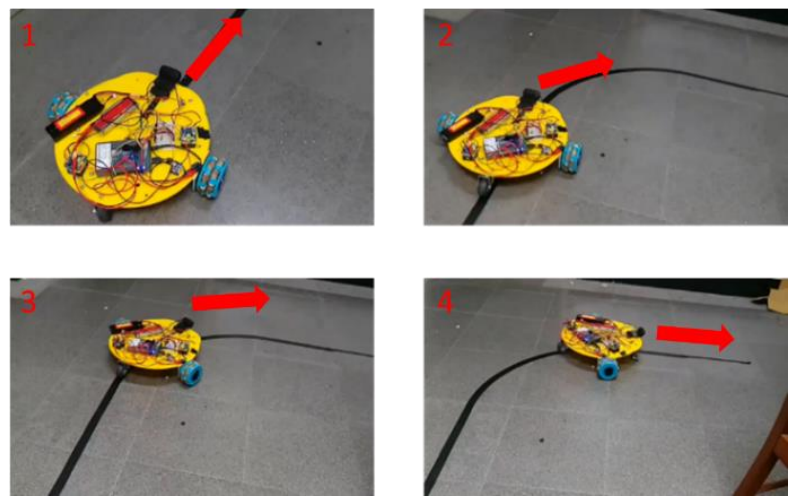

Fig.9 Vision line tracking test
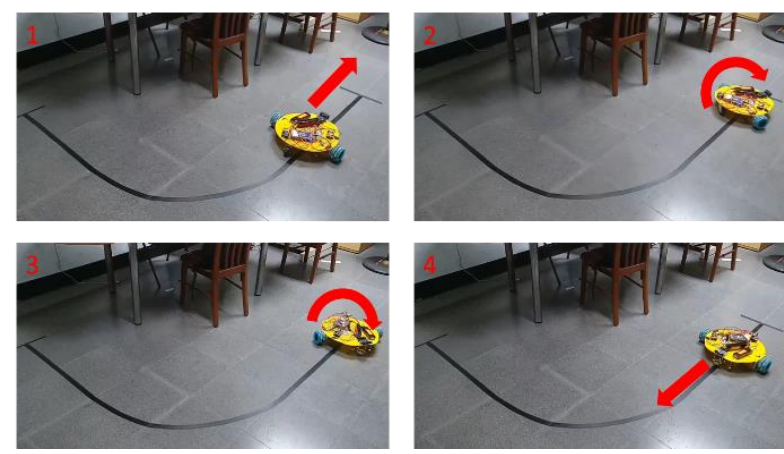

Fig.10 Search for horizontal lines and return 


\subsection{Sign Identification Demo}

In this case, we integrate perspective calibration for geometric matching and vision line tracking demo. We use turn signs to instruct the vision guided vehicle to move and turn. If the vehicle drives to the end of the line, it will turn around and back to the line. However, in this experiment, we use the computer to conduct image processing but not MyRIO, that because the MyRIO have not enough frame rate for real time control when doing a lot of image processing. Although we have completed the integration of perspective calibration and vision line tracking, there is still much need for improvement in programming. The conclusion is that the machine vision approach can be used for lane navigation, and the accuracy of template matching is improved by using the perspective calibration.
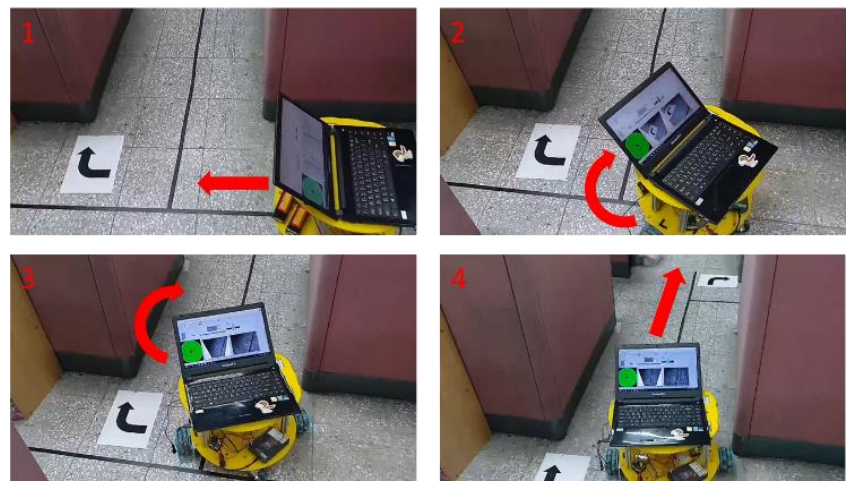

Fig.11 sign identification test

\section{Discussion and Conclusion}

\subsection{Discussion}

Automated guided vehicles and machine vision recognition play an extremely important role in the development of Industry 4.0. Inspired by this, we developed a vision guided vehicle that integrates two technologies. However, we still have problems with machine vision misjudgment in this experiment, so we hope that in the future, we can use the technology such as neural network to improve the machine vision recognition. In addition, although the recognition of the object distance is made more accurate by using the perspective calibration, this function has not been used in the automatic guided vehicle, which is one of the goals that we still have to work hard in the future.

\subsection{Conclusion}

In this study, the homography matrix and perspective calibration are developed to promote the precision of the geometric matching as well as the distance of the object. An experimental prototype is established to validate the automatic guided vehicle by conducting experiments for vision line tracking and sign distinguish. Although this system is still not an effective system to application, the experiment provides the foundation for set up an automatic guided vehicle. Despite the result is still primitive, the automatic guided vehicle has been improved by taking advantage from machine vision system. In summary, by integrating perspective calibration, automatic guided vehicle, and image processing, a preliminary system with comprehensive schemes has been developed, which is the goal of this work. A more comprehensive study involving automatic control is currently underway. It is believed that the prototype established in this study should be useful for either automatic guided vehicle or developing methods for machine vision.

\section{Acknowledgment}

This paper is supported by the Ministry of Science and Technology (MOST) of Taiwan under contract No. 1072221-E-006-129 and No. MOST 105-2221-E-006 -100 MY3 and No. MOST 105-2221-E-006 -074 -MY3.

\section{References}

(1) Z. Kim, "Robust lane detection and tracking in challenging scenarios," IEEE Transaction on Intelligent Transportation System., vol. 9, no. 1, pp. 16-26, Mar. 2008

(2) S. Feng, T. Wang, H. Huang, and L. Chen , "An Image Mosaic Method Based on Deviation Splitting and Transferring for Vehicle Panoramic System," International Conference on Computer Vision Systems, Springer International Publishing, pp. 218-227, 2017.

(3) K. Kanatani, "Optimal homography computation with a reliability measure," Proc. IAPR Workshop Machine Vision Applications, pp. 426-429,1998

(4) S. Butdee and A. Suebsomran, "Automatic guided vehicle control by vision system," Industrial Engineering and Engineering Management, pp. 694697,2009

(5) Y. S. Chien, A Homogrphy Transformation-based Car Surround View System, Master's Thesis, Department of Engineering Science, National Cheng Kung University, pp5-8,2016 